\title{
Estudo de alguns cenários climáticos para o Nordeste do Brasil
}

\author{
David N. dos Santos' ${ }^{1}$ Vicente de P. R. da Silva' ${ }^{1}$ Francisco de A. S. Sousa' $\&$ Roberta A. e Silva'
}

\begin{abstract}
RESUMO
Propôs-se, neste trabalho, avaliar as condições climáticas atuais da região Nordeste do Brasil e elaborar os cenários climáticos para os anos de 2050 e 2100. Utilizaram-se as séries temporais da temperatura do ar média correspondentes ao período de 1961 a 2007, e as médias climatológicas de precipitação pluvial de 89 localidades no Nordeste do Brasil. 0 teste não-paramétrico de Mann-Kendall foi empregado para avaliar o nível de significância das tendências das séries temporais da temperatura do ar média e o "software" SEVAP, para obtenção da evapotranspiração potencial anual e dos índices de aridez, umidade e hídrico. 0 s resultados obtidos indicam tendências crescentes nas séries temporais de temperatura do ar, estatisticamente significativas pelo teste de Mann-Kendall, tanto para o período estudado como para os cenários de 2050 e 2100. As maiores taxas de aumento das temperaturas do ar médias anuais foram encontradas para a situação climática atual e para o cenário do ano de 2050, e menor aumento de seus valores entre os cenários de 2050 e 2100. 0 s índices de aridez e hídrico indicam aumentos enquanto 0 índice de umidade mostra redução nos cenários estudados, principalmente na parte semiárida da região.
\end{abstract}

Palavraschave: evapotranspiração potencial, índice de aridez, teste de Mann-Kendall

\section{Study of some climate scenarios for the northeastern region of Brazil}

\begin{abstract}
The main objective of this study is to assess the current climatic conditions in northeastern region of Brazil and to obtain some climatic scenarios for the years 2050 and 2100. Time series of mean air temperature and rainfall from 1961 to 2007 period and 89 stations in the study region were used. The non-parametric test of Mann-Kendall was used to assess the statistical significance level of the analyzed time series. Also, the software SEVAP was used to determine potential evapotranspiration, humidity index, aridity index and water index. Results showed an increasing trend in the time series of air temperature, which were statistically significant by the Mann-Kendall test, for the current period and for the 2050 and 2100 scenarios. Mean air temperature has its highest rate for the current period and for the 2050 scenarios and a reduced increase between the 2050 and 2100 scenarios. The aridity and water indexes indicated an increase while the humidity index showed a reduction for two analyzed scenarios in northeastern region of Brazil, principally in the semiarid area.
\end{abstract}

Key words potencial evapotranspiration, arid index, Mann-Kendall test 


\section{INTRODUÇÃO}

O interesse da comunidade científica pelo estudo da variabilidade e mudanças climáticas vem aumentando de forma bastante acentuada nas últimas décadas, devido principalmente às suas consequências para a humanidade e a biodiversidades dos sistemas naturais. Muitos estudos têm sido desenvolvidos em várias partes do mundo visando compreender melhor e mitigar os efeitos desses processos (Arnell, 1999; Cunha et al., 2002; Silva, 2004; Modarres \& Silva, 2007; Ma et al., 2008; Todisco \& Vergni, 2008).

Investigações sobre a variabilidade e a mudança do clima usam a temperatura média global da superfície para estabelecer o grau e o significado das mudanças do clima durante o último século (Silva et al., 2006). A razão disto é a expectativa de que a temperatura média global responda à mudança da forçante radiativa associada aos gases do efeito estufa e aerossóis da atmosfera. As mudanças climáticas resultam das alterações das variáveis meteorológicas, como a precipitação pluvial, temperatura do ar, vento, radiação solar e umidade relativa do ar, ou seja, das variáveis representativas do clima que, ao longo do tempo, geram modificações nos ecossistemas naturais. A temperatura média global também é um indicador simples da variabilidade interna do clima em simulações com modelos e em observações. Também, geralmente ela é usada como o índice mais simples de variabilidade e de mudança do clima global (Back, 2001; Braganza et al., 2003). Considerando o cenário de aumento da temperatura do globo, outros impactos ambientais estão ocorrendo, como consequência da mudança no clima, tais como a redução da cobertura de gelo nos polos, o aumento do nível do mar devido ao degelo das geleiras, eventos extremos de secas e chuvas e mudanças no regime de precipitação pluvial em diferentes regiões do globo. Esses são alguns exemplos de desastres naturais que poderão ter impactos decorrentes das mudanças climáticas devido à grande vulnerabilidade associada aos sistemas naturais (Marengo \& Valverde, 2007).

Atualmente, diversos estudos sobre mudanças climáticas no Brasil se juntam a essas pesquisas uma vez que o clima do País também tem experimentado alterações, em particular na região semiárida. Secas severas e enchentes são exemplos reais que servem de advertência para o futuro.

A região Nordeste do Brasil, apesar de chover tanto quanto em muitas outras regiões do mundo, em particular na parte semiárida, é periodicamente afetada pela ocorrência de secas com perdas parciais ou totais na agropecuária, além de comprometer o abastecimento de água devido principalmente à irregularidade da estação chuvosa na região, com predominância de chuvas intensas e de curta duração (Silva et al., 1998). Estudos recentes indicam que esta região vem sofrendo com fortes variabilidades climáticas (Pimenta et al., 1998; Silva, 2004; Sousa Júnior, 2006). Assim, grande parte do semiárido brasileiro, onde a agricultura não-irrigada já é uma atividade marginal, se torna ainda mais inadequada para a prática da agricultura de subsistência.

Yamazaki \& Rao (1977), observando imagens de satélite, sugeriram que a importância dos distúrbios de leste, também encontrados na literatura como Ondas de Leste (OL), são frequentemente relacionados com a precipitação pluvial que ocorrem no leste do Nordeste do Brasil. Chan (1990) observou que essas ondas se propagam do Oceano Atlântico em direção ao continente, durante o outono/inverno (maio a agosto). Outro fator que favorece as chuvas no norte, nordeste e oeste da região, é a presença dos Vórtices Ciclônicos de Altos Níveis (VCAN). Esses vórtices são observados nos meses de setembro a abril, tendo maior frequência em janeiro. Neste contexto e se considerando a importância das mudanças climáticas para o futuro do planeta, o objetivo deste trabalho é avaliar as condições climáticas atuais e elaborar alguns cenários climáticos, com base em observações de superfície, para a referida região.

\section{MATERIAL E MÉTODOS}

\section{Localização geográfica e climatologia da região}

O Nordeste brasileiro se situa aproximadamente entre as latitudes de $1^{\circ}$ e $18^{\circ} 30^{\prime} \mathrm{S}$ e longitudes de $34^{\circ} 20^{\prime}$ e $48^{\circ} 30^{\prime}$ W; compreende nove estados da Federação e ocupa uma área superior a 1,5 milhão de $\mathrm{km}^{2}$; o clima semiárido corresponde a $60 \%$ de sua área total com precipitação pluvial média anual inferior a $500 \mathrm{~mm} \mathrm{ano}^{-1}$ em algumas localidades. A variabilidade interanual da distribuição de chuvas no Nordeste do Brasil (NEB), tanto em escala espacial quanto temporal, está intimamente relacionada com as mudanças nas configurações de circulação atmosférica de grande escala e com a interação oceano-atmosfera no Pacífico e no Atlântico (Molion \& Bernardo, 2002).

Dentre os principais mecanismos físicos produtores de chuva na região, se destaca a Zona de Convergência Intertropical (ZCIT), pela influência direta na estação chuvosa no norte do NEB. Este sistema chega à sua posição mais ao sul do NEB nos meses de março e abril, com a ocorrência da máxima precipitação, com níveis entre 1000 e 1200 mm/ano (Hastenrath \& Heller, 1977). Por outro lado, o sul do NEB tem, como principal mecanismo de precipitação, os sistemas frontais estacionários alimentados pela umidade proveniente do Atlântico Sul, que definem a Zona de Convergência do Atlântico Sul (ZCAS), os sistemas pré-frontais, convecção local e brisas marítimas e terrestres. Nesta região os índices pluviométricos variam de $300 \mathrm{~mm} \mathrm{ano}^{-1}$, no interior, a mais de $3000 \mathrm{~mm} \mathrm{ano}^{-1}$ no litoral. Tal regime de chuvas é semelhante àquele que ocorre na parte norte da região Sudeste do Brasil (Molion \& Bernardo, 2002). O máximo de precipitação pluvial no sul da região está associado à penetração de frentes frias vindas do sul que alcançam latitudes mais baixas nos meses de novembro a fevereiro (Alves \& Kayano, 1991). Esta região também tem, como principal característica climática, a alta variabilidade espacial e temporal da precipitação pluvial, determinada sobretudo por padrões de grande escala da circulação geral da atmosfera, associada às características locais de topografia (Nobre \& Molion, 1988).

\section{Dados utilizados}

Neste estudo se utilizaram dados observados na superfície de temperatura do ar (médias mensais), obtidos junto ao 
Instituto Nacional de Meteorologia - INMET, no período compreendido entre 1961 e 2007, além de dados climatológicos de precipitação pluvial, cedidos pela Unidade Acadêmica de Ciências Atmosféricas - UACA, da Universidade Federal de Campina Grande - UFCG, no período de 1911 a 1990. O estudo compreende 89 estações meteorológicas espacialmente bem distribuídas nos nove estados do Nordeste brasileiro.

\section{Procedimentos metodológicos}

As séries temporais de dados foram submetidos a uma análise de consistência, eliminando-se aquelas com dados faltosos e com menos de 30 anos de observações. O programa computacional, denominado Sistema de Estimativa de Evapotranspiração (SEVAP) (Silva et al., 2005) foi utilizado para se obter o balanço hídrico climatológico e a classificação climática, segundo Thornthwaite (1948) para cada estação analisada; desta se obtiveram o índice de aridez (Ia), o índice hídrico (Ih) e o índice de umidade (Iu), e a evapotranspiração potencial anual (ETP), utilizando-se a capacidade de água disponível de acordo com as características de cada estação, e a precipitação pluvial média mensal para cada posto analisado; posteriormente, mapas temáticos dessas variáveis foram elaborados, correspondentes aos cenários atuais e para os anos de 2050 e 2100 . Na elaboração desses mapas lançou-se mão do GrADS (Grid Analysis and Display System) em associação com o método de interpolação de Barnes (1964).

\section{Análise estatística}

O teste de Mann-Kendall é um teste não-paramétrico (Mann, 1945; Kendall, 1975) que consiste em comparar cada valor da série temporal com os valores restantes, sempre em ordem sequencial; é contado o número de vezes em que os termos restantes são maiores que o valor analisado. A estatística S é obtida pela soma de todas as contagens, como segue: em que o sinal $\left(\mathrm{x}_{\mathrm{i}}-\mathrm{x}_{\mathrm{j}}\right)$ é obtido da seguinte forma:

$$
\mathrm{S}=\sum_{\mathrm{i}=2}^{\mathrm{n}} \sum_{\mathrm{j}=1}^{\mathrm{i}-1} \operatorname{sinal}\left(\mathrm{x}_{\mathrm{i}}-\mathrm{x}_{\mathrm{j}}\right)
$$

A estatística S tende para a normalidade quando n é gran-

$$
\text { Sinal }=\left\{\begin{array}{rrr}
1 & \text { se } & \left(x_{i}-x_{j}\right)>0 \\
0 & \text { se } & \left(x_{i}-x_{j}\right)=0 \\
-1 & \text { se } & \left(x_{i}-x_{j}\right)<0
\end{array}\right.
$$

de, com média E(S) e variância $\operatorname{Var}(\mathrm{S})$ definidas como seguem:

$$
\mathrm{E}(\mathrm{S})=0
$$

$$
\operatorname{Var}(S)=\frac{\mathrm{n}(\mathrm{n}+1)(2 \mathrm{n}+5)-\sum_{\mathrm{i}=1}^{\mathrm{q}} \mathrm{t}_{\mathrm{p}}\left(\mathrm{t}_{\mathrm{p}}-1\right)\left(2 \mathrm{t}_{\mathrm{p}}+5\right)}{18}
$$

em que $\left(\mathrm{t}_{\mathrm{p}}\right)$ é número de dados com valores iguais em certo grupo (pth) e q o número de grupos contendo valores iguais na série de dados em um grupo p. O segundo termo da Eq. 4 representa um ajuste para a variância os dados.
O teste estatístico parametrizado $\left(\mathrm{Z}_{\mathrm{MK}}\right)$ é dado por:

$$
\mathrm{Z}_{\mathrm{MK}}= \begin{cases}\frac{\mathrm{S}-1}{\sqrt{\operatorname{Var}(\mathrm{S})}} & \mathrm{S}>0 \\ 0 & \mathrm{~S}=0 \\ \frac{\mathrm{S}+1}{\sqrt{\operatorname{Var}(\mathrm{S})}} & \mathrm{S}<0\end{cases}
$$

A presença de tendência estatisticamente significativa na série temporal é avaliada através do valor de Z; esta estatística é usada para testar a hipótese nula de que nenhuma tendência existe. $\mathrm{O}$ valor positivo de $\mathrm{Z}_{\mathrm{MK}}$ indica tendência crescente. Para testar qualquer tendência constante, crescente ou decrescente para um nível significante de p, a hipótese nula é rejeitada se o valor absoluto de $Z$ é maior que $Z_{1-p / 2}$, que é obtido na tabela da distribuição normal. Neste trabalho se aplicaram os níveis de significância de p=0,01 e 0,05.

\section{RESULTADOS E DISCUSSÃO}

\section{Temperatura do ar média}

Na situação climática atual, a temperatura do ar média anual apresentou tendências de aumento em quase todas as estações estudadas. Na Figura 1B se encontra a espacialização das tendências da temperatura do ar média no Nordeste do Brasil (1961-2007). Conforme a distribuição apresentada na Figura 1C, as regiões sul dos Estados de Alagoas e Sergipe, oeste e nordeste da Bahia, sul/sudoeste do Piauí e grande parte do Maranhão, apresentam tendências crescentes na temperatura do ar estatisticamente significativas a níveis de 1 e $5 \%$ de probabilidade pelo teste de Mann-Kendall. Dentre as estações dessa grande área, apenas três indicaram tendência de redução (Maceió, AL, Guaratinga e Itiruçú, BA), com significância estatística a nível de 5\% de probabilidade. Silva (2004) também encontrou tendências positivas da temperatura do ar estatisticamente significativas em várias localidades do Nordeste do Brasil. Na distribuição de temperatura do ar média (Figura 1A) se encontram temperaturas médias do ar variando entre 22 a $25^{\circ} \mathrm{C}$ porém, no cenário para 2050 (Figura 1D), as temperaturas médias do ar se dispõem entre 27 e $28^{\circ} \mathrm{C}$ em grande parte dos Estados do Maranhão, Piauí, Ceará e Rio Grande do Norte, enquanto na parte centro-sul do Nordeste do Brasil as temperaturas médias do ar variaram entre 21 e $26^{\circ} \mathrm{C}$. Para o cenário de 2100 (Figura 1E) a Tmed variou entre 27 a $31^{\circ} \mathrm{C}$ no NEB; apenas na região sul do Nordeste do Brasil, tanto nos mapas de distribuição espacial de temperatura média do ar quanto nos mapas dos cenários de temperaturas máximas e mínimas, se constatam os menores valores de temperaturas do ar. Analisando a Tmed nas capitais do Nordeste brasileiro, destaca-se a estação de Teresina, PI, com o menor acréscimo de temperatura média do ar, de $0,0144{ }^{\circ} \mathrm{C}$ ano-1 ${ }^{-1}$, resultando um aumento de $0,62{ }^{\circ} \mathrm{C}$ para o cenário de 2050 e de $1,34{ }^{\circ} \mathrm{C}$ para o cenário de 2100 . Por outro lado, para São Luis, MA, a tendência é de $0,0145^{\circ} \mathrm{C}$ ano ${ }^{-1}$; logo, bastante semelhante da encontrada para Teresina. Esses resultados 
A.

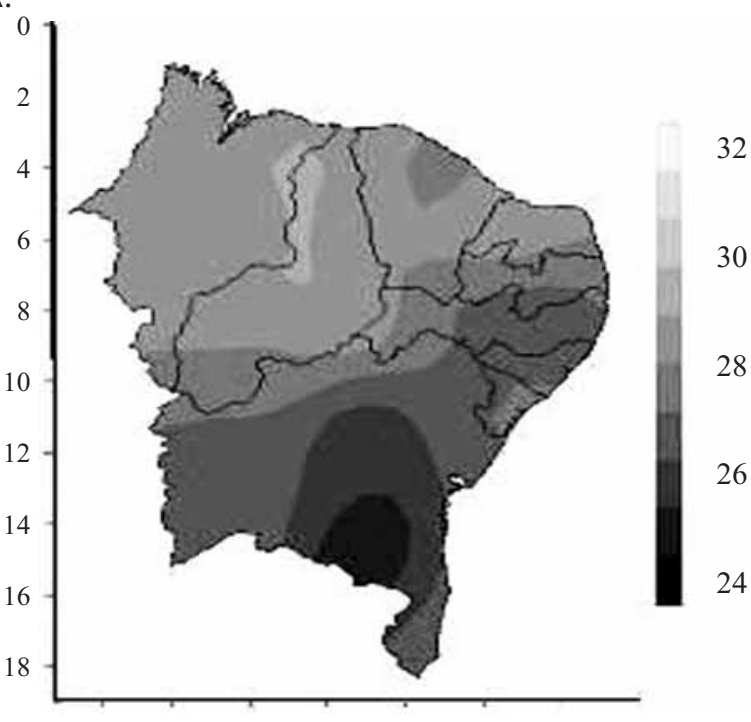

C.

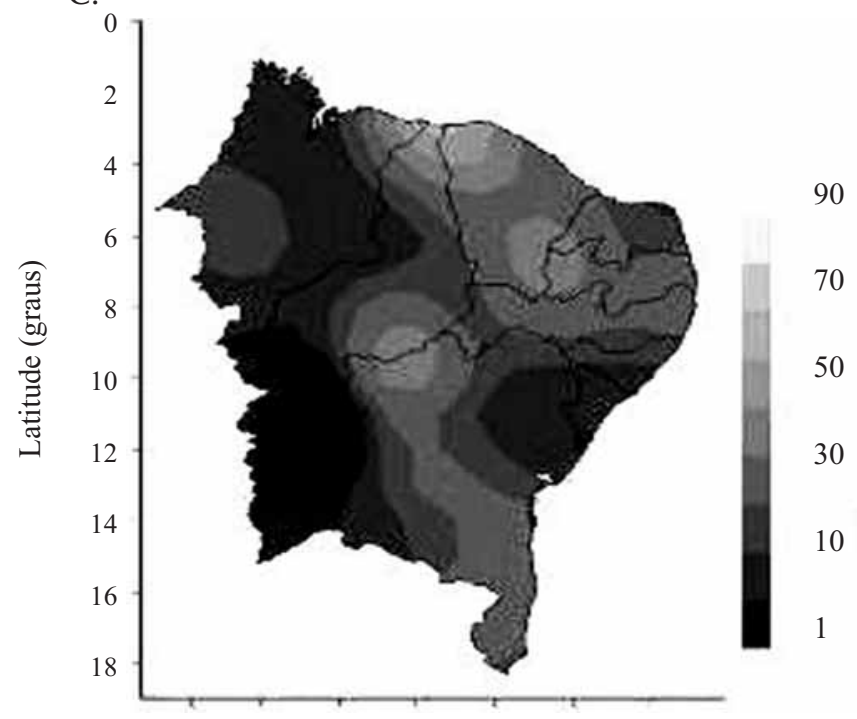

E.

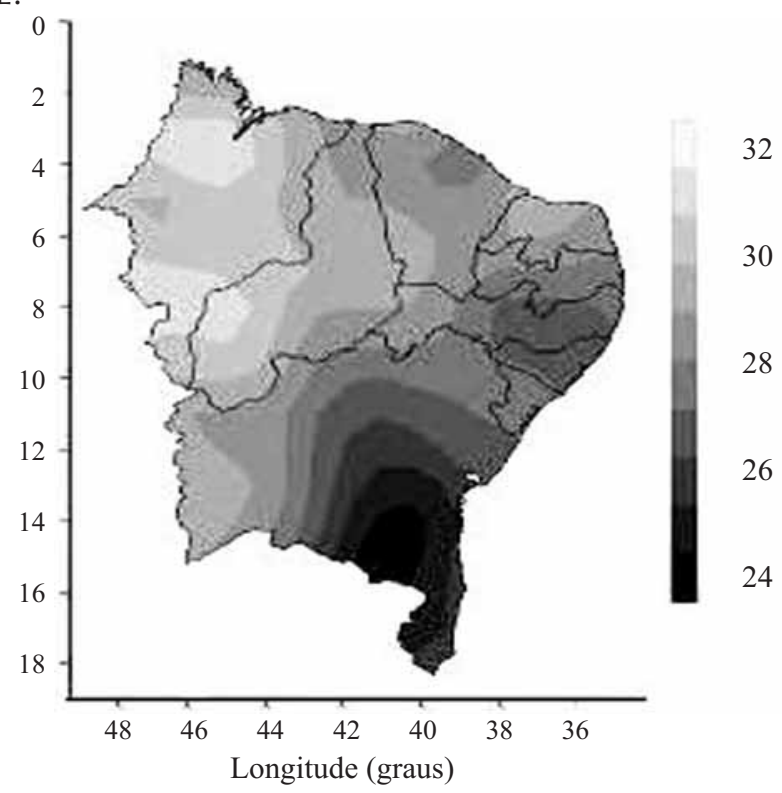

B.

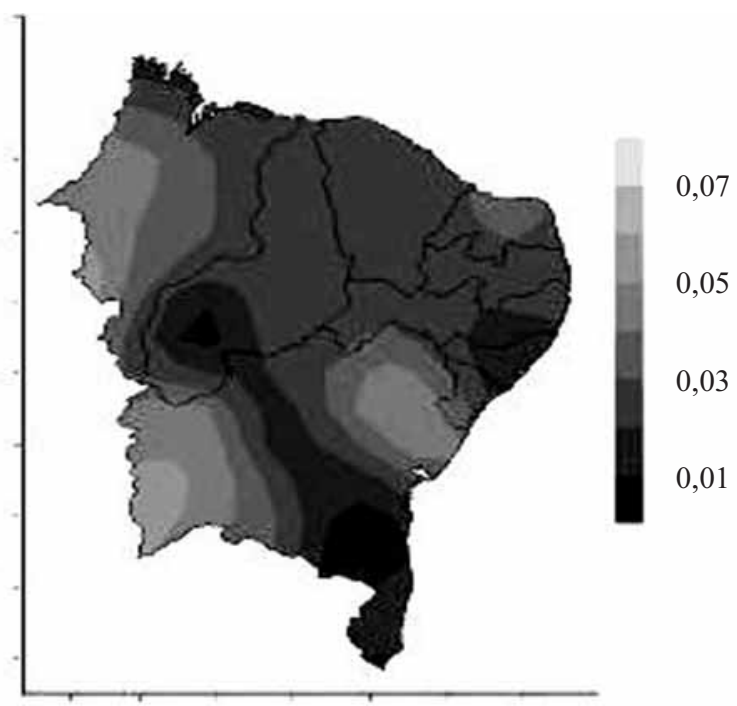

D.

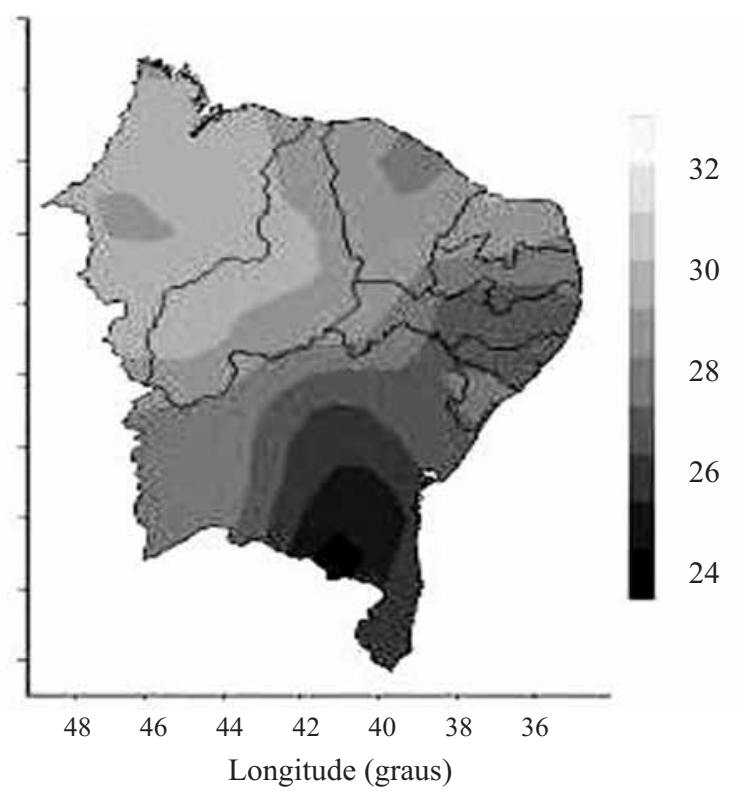

Figura 1. Distribuicão espacial da temperatura do ar média anual do período de 1961 a 2007 (A), tendência temporal (B), nível de significância da tendência (C), cenário para o ano de 2050 (D) e cenário para o ano de 2100 (E) no Nordeste do Brasil 
sugerem que as previsões apresentadas para o NEB no relatório AR4 do IPCC (2007) superestimam as tendências dos dados observados na região de estudo.

\section{Evapotranspiração potencial anual média}

Observou-se aumento na evapotranspiração potencial média (ETPmed) do ar nos cenários de 2050 e de 2100 em relação à situação climática atual (Figura 2). A ETPmed no período analisado indica o tipo de clima com característica megatérmico em grande parte da região estudada, com valores variando entre 1100 a $1400 \mathrm{~mm}$ por período nos Estado da Bahia, Sergipe, Alagoas, Pernambuco e Paraíba (Figura 2A). Na região que compreende os Estados do Maranhão, Piauí, Ceará e Rio Grande do Norte, são encontrados valores de ETPmed entre 1500 e $1700 \mathrm{~mm}$ por período.

Os resultados do cenário para 2050 indicaram aumento na ETPmed em relação à situação atual, com valores entre 1500 e $1800 \mathrm{~mm}$ por período (Figura 2B) e, no cenário de 2100, apenas pequenas áreas ao sul do NEB apresentam valores entre 1100 a $1400 \mathrm{~mm}$ por período enquanto o restante da região mostra valores entre 1500 a $2000 \mathrm{~mm}$ por período (Figura 2C).

As estações com as maiores taxas de aumento de ETPmed entre a situação atual e o cenário para o ano de 2050, foram Bom Jesus do Piauí, PI, com 327,7 mm por período e Tauá, CE, com 317,7 mm por período; já com relação à taxa de aumento entre o cenário de 2050 e 2100, se destacam as estações de Campina Grande, PB, com 269,6 mm por período e Caracol, PI, com 268,4 mm por período. Por outro lado, as estações que apresentaram os maiores acréscimos de ETPmed entre a situação média atual da região e o cenário para o ano de 2100, foram Barbalha, CE, e Caracol, PI, com 553,2 e $547,8 \mathrm{~mm}$ por período, respectivamente. Analisando todas as estações com relação aos cenários analisados, verifica-se uma diferença maior de aumento de ETPmed atual para o cenário de 2050 que entre os cenários de 2050 e 2100. O clima no Nordeste do Brasil é essencialmente megatérmico (ETP > $1.140 \mathrm{~mm}$ ), de acordo com a classificação climática de Thornthwaite (1948), com expressiva área mesotérmica no sul da região $(997 \mathrm{~mm}<\mathrm{ETP}>1.140 \mathrm{~mm}$ ); entretanto, para o cenário de 2050 a área mesotérmica é bastante reduzida e, para o cenário de 2100 , toda a região se torna praticamente megatérmica.

\section{Índice de aridez médio}

A distribuição espacial do índice de aridez anual médio (Iamed) no Nordeste do Brasil para a situação atual e para os cenários de 2050 e 2100, é evidenciada na Figura 3 verificando-se aumento no Iamed entre a situação atual e os cenários estudados (Figura 3A, B e C). Pode-se observar, ainda, que em grande parte da região há valores de Iamed entre 30 e 60, com exceção do extremo norte do Estado do Maranhão, onde se encontram valores entre 20 e 25. No sul da Bahia estão os menores valores desse índice, tanto para a situação atual quanto para os cenários estudados (Figura 3A). De acordo com o cenário de 2050 (Figura 3B), pode-se destacar o aumento nos valores do Iamed até mesmo acima 60, na região do sertão nordestino. Na Figura 3C, que corresponde ao cenário para
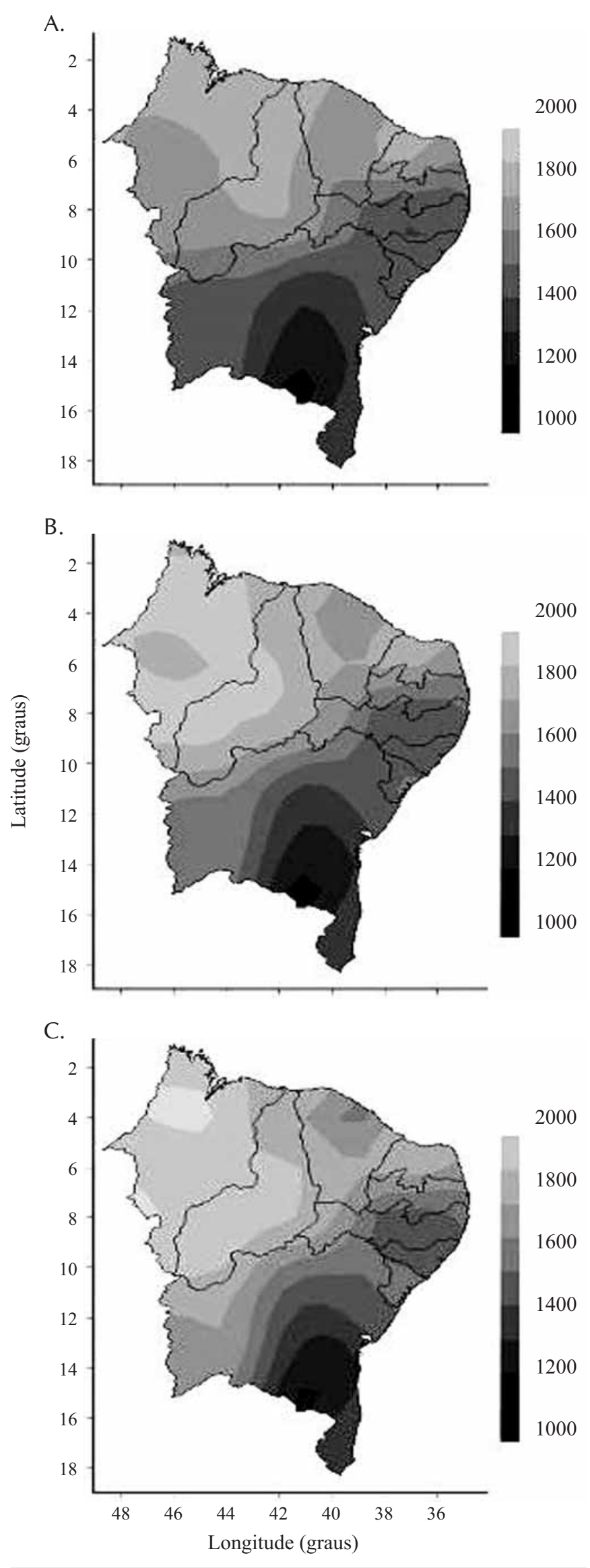

Figura 2. Evapotranspiração potencial anual média do período de 1961 a 2007 (A), cenário para o ano de 2050 (B) e cenário para 0 ano 2100 (C) no Nordeste do Brasil 

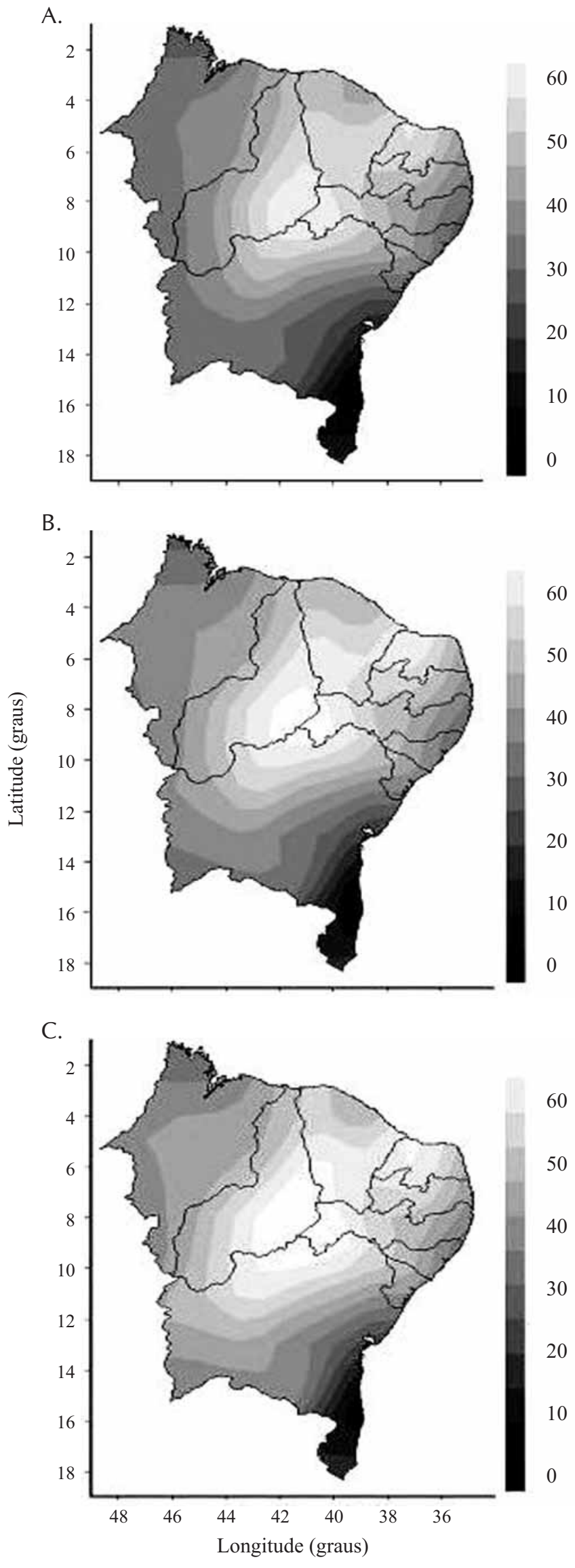

Figura 3. Distribuição espacial do índice de aridez médio anual do período de 1961 a 2007 (A), cenário para o ano de 2050 (B) e cenário para 0 ano de 2100 (C) no Nordeste do Brasil
2100, observa-se que a região central do NEB exibe valores de Iamed acima de 60 e, em outras partes da região, valores entre 30 e 60. A região sul do Estado da Bahia mostra os menores valores de Iamed, tanto na situação atual quanto para os cenários estudados. A maior diferença entre os valores de Iamed da situação atual na região e o cenário para 2050 se encontra em Caracol, PI (10,7) e entre os cenários de 2050 e 2100 em Campina Grande, PB (9,8). A estação de Campina Grande também apresentou a maior diferença entre os valores de Iamed entre a média da situação atual e o cenário para o ano de 2100 (19,7). Resultados revelam reduções do Iamed em todos os cenários, porém em menor escala, especificamente em Itiruçu, BA, e Maceió, AL. Em 64\% das estações analisadas se deu o maior aumento do Iamed entre a situação atual e o cenário para o ano de 2050; já em 28\% o maior aumento ocorreu entre os cenários de 2050 e 2100. Esta análise é particularmente válida para a região semiárida do Nordeste do Brasil, haja vista que nem todas as microrregiões do Nordeste do Brasil apresentam aumento de temperatura do ar ao longo do tempo.

\section{Índice de umidade média}

A Figura 4 exibe a distribuição espacial do índice de umidade média anual (Iumed) para a situação atual no Nordeste do Brasil e para os cenários de 2050 e 2100 . Verificase redução no Iumed entre a situação atual e os cenários analisados indicando um ressecamento ambiental acentuado para os anos de 2050 e 2100. Analisando a situação atual do Iumed (Figura 4A) pode-se destacar duas regiões que apresentam os maiores valores: o extremo norte do Estado do Maranhão, com valores variando entre 40 e 50, e o sul do Estado da Bahia, com valores variando de 40 a 70 . Esses resultados concordam com os observados para Iamed nessas áreas (Figura 3A). Com base nos cenários para 2050 (Figura 4B) e para 2100 (Figura 4C) verifica-se que não houve diferenças acentuadas nos valores do Iumed. Nas regiões central e sul do Estado do Maranhão esses valores variaram entre 0 e 10 e, no litoral leste do Nordeste, os valores de Iumed ficaram entre 10 e 20. A estação de Maceió, AL, foi a única que apresentou acréscimo de Iumed entre o período atual e o cenário para 2050 (6,0) e entre os cenários de 2050 e $2100(7,2)$. Por outro lado, as estações que apresentaram os maiores decréscimos de Iumed foram Carolina, MA (entre o período atual e cenário para 2050 foi de -14,0) e Carinhanha, BA (entre os cenários de 2050 e 2100 foi de -9,4).

\section{Índice hídrico médio}

Os mapas dos índices hídricos anuais médios (Ihmed), para a situação atual e para os cenários estudados, são exibidos na Figura 5; verificou-se diminuição razoável de Ihmed, indicando aumento na deficiência hídrica no NEB. Os resultados também revelam tipos climáticos subúmido, seco subúmido e semiárido na situação climática atual e para os cenários de 2050 e 2100 (Figura 5), sendo que para o cenário de 2100 ocorre a maior expansão do Ihmed, atingindo até o sudeste do Estado do Maranhão; apenas para a situação atual é possível encontrar o tipo de clima úmido, precisamente no extremo sul da Bahia (Figura 5A). A estação de 
A.
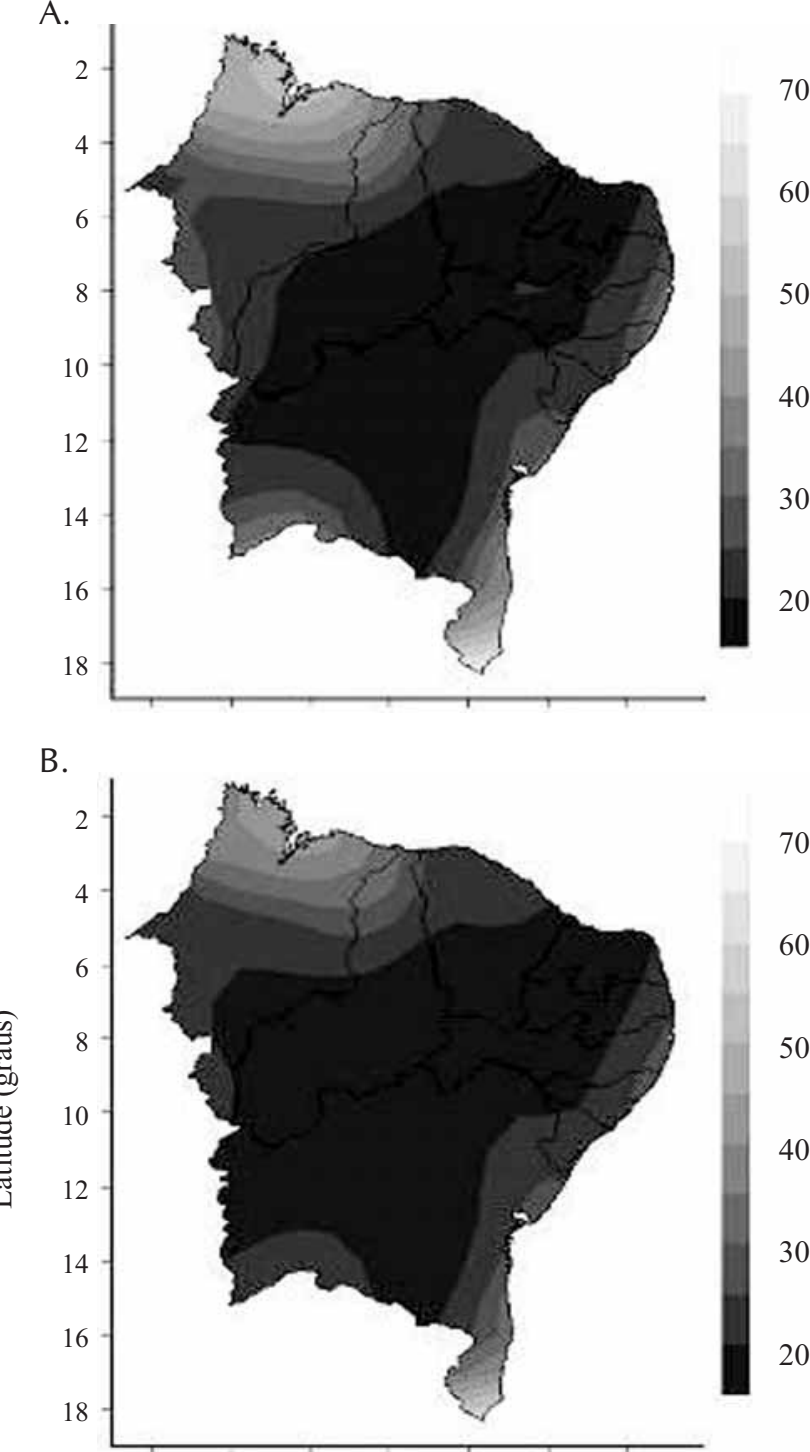

C.

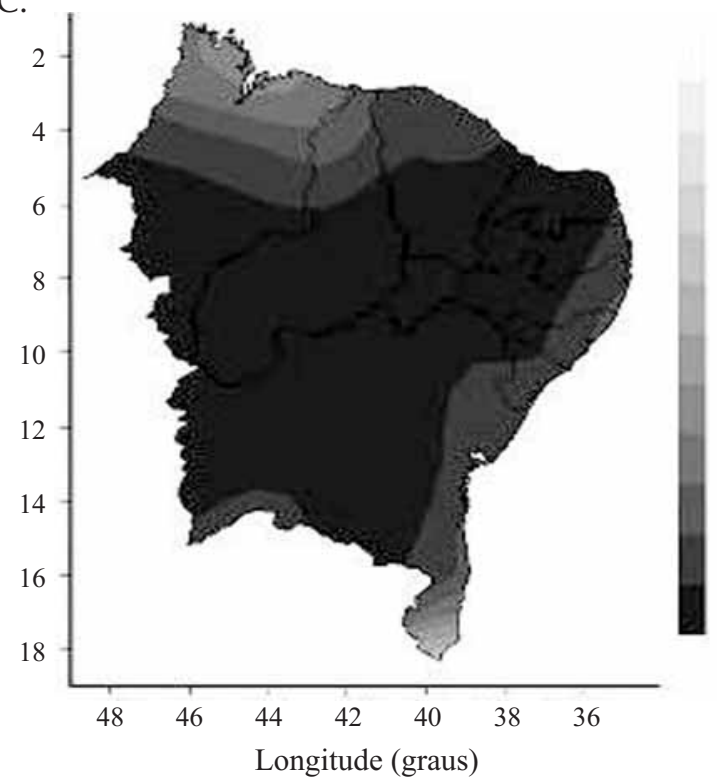

Figura 4. Distribuição espacial do índice de umidade médio anual do período de 1961 a 2007 (A), cenário para 0 ano de 2050 (B) e cenário para 0 ano de 2100 (C) no Nordeste do Brasil
A.
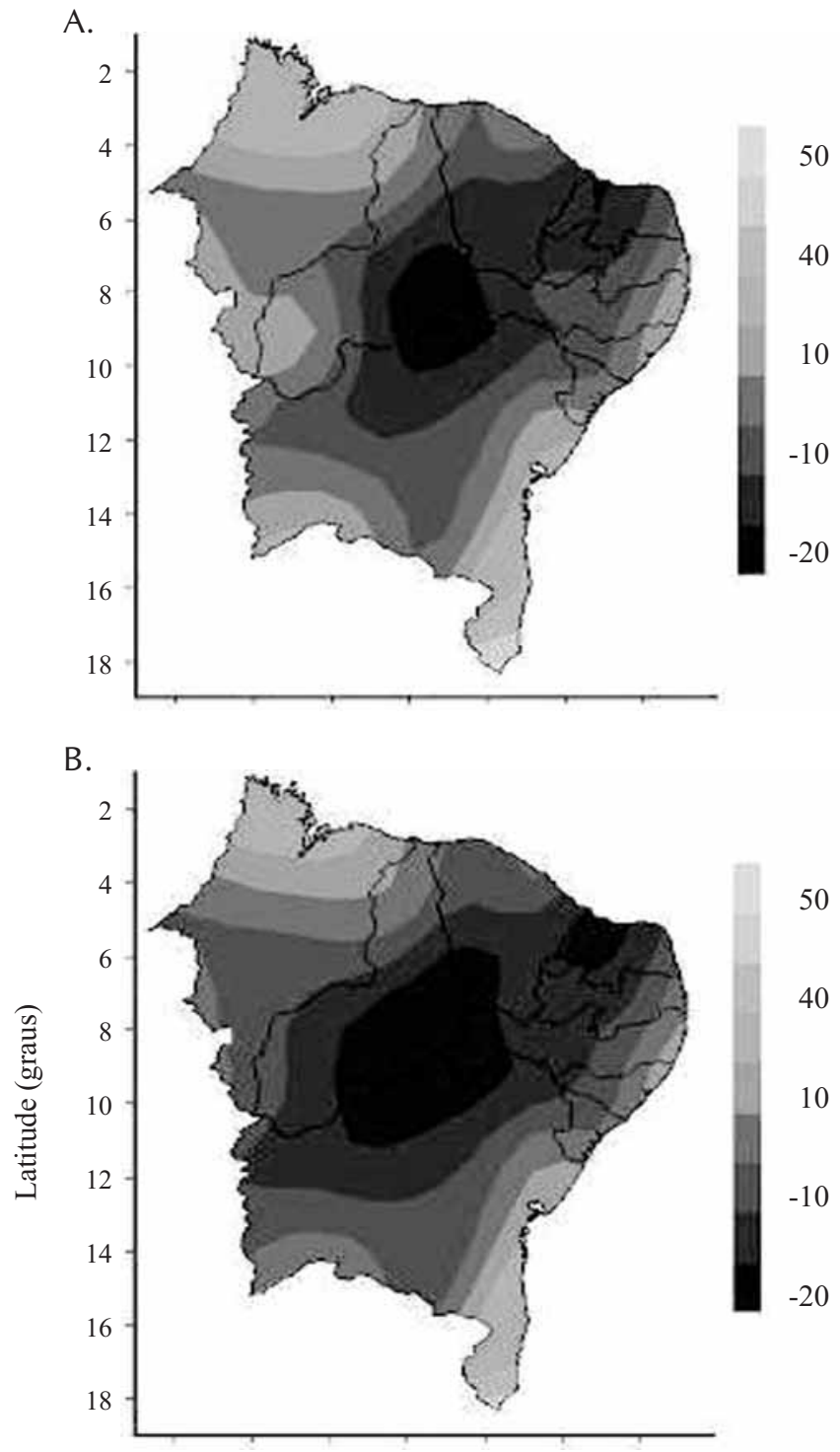

C.

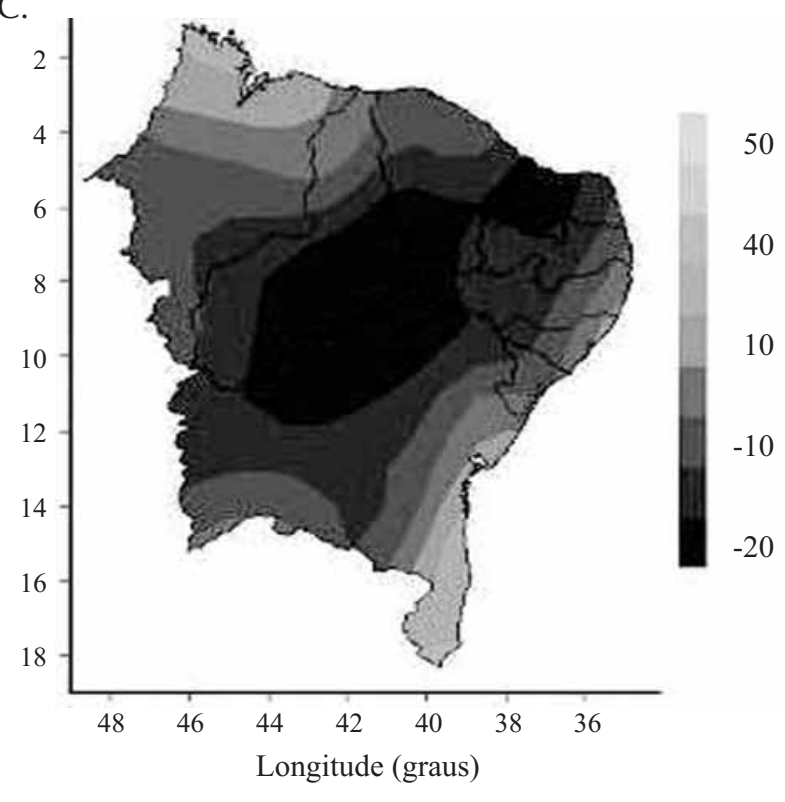

Figura 5. Distribuição espacial do índice hídrico anual médio do período de 1961 a 2007 (A), cenário para o ano de 2050 (B) e cenário para 0 ano de 2100 (C) no Nordeste do Brasil 
Maceió, Al, indicou as maiores diferenças no aumento do Ihmed para o período compreendido entre a situação atual e ocenário para $2050(7,8)$ e entre os cenários de 2050 e 2100 $(9,0)$. Entre a situação atual e o cenário para 2050, a estação de Bom Jesus do Piauí, PI, apresentou a maior redução no valor de Ihmed $(-54,1)$ enquanto entre os cenários de 2050 e 2100 a estação de Carinhanha, BA, mostrou redução de $-10,6$ no Ihmed. No período compreendido entre a situação atual e o cenário para 2050 ocorreu a maior redução no Ihmed, em 75,2\% das estações; já entre os cenários de 2050 e 2100 o maior aumento de déficit hídrico foi de apenas 20,2\% das estações estudadas.

Os resultados obtidos neste trabalho indicam que a temperatura do ar, evapotranspiração potencial e índice de aridez, têm tendências de aumento e os índices de umidade e hídrico de redução em todo o Nordeste do Brasil nos cenários estudados (2007-2050 e 2050-2100) quando comparados com o período de estudo (1961-2007). Hertig \& Jacobeit (2008), usando dois modelos de circulação geral global (ECHAM4/OPYC3 e HadCM3), observaram que, em todos os meses do ano e em toda a área Mediterrânea, a temperatura do ar aumentou no período de 2071-2100 em relação ao período de 1990-2019. Ainda de acordo com a Figura 5, a área semiárida (índice hídrico entre -20 e -40) da região Nordeste do Brasil cresceu substancialmente da situação atual para o cenário de 2050, mais ainda para o cenário de 2100, chegando a atingir, na porção central da região, valores do índice hídrico compatíveis para regiões áridas (índice hídrico entre -40 e -60).

\section{CONCLUSÕES}

1. A região Nordeste do Brasil apresenta tendências estatisticamente significativas de aumento nas temperaturas médias do ar. As maiores taxas de aumento dessa variável foram encontradas entre a situação climática atual e o cenário para o ano de 2050; já entre os cenários climáticos de 2050 e 2100 a região apresentou o menor aumento de temperatura do ar.

2. A demanda evaporativa representada pela evapotranspiração potencial, também aponta tendências crescentes em toda a região estudada, para a situação climática atual e para os dois cenários analisados.

3. O índice de aridez indicou aumento e os índices de umidade e hídrico mostraram redução para os cenários estudados, em toda a região do Nordeste do Brasil, principalmente na parte semiárida, indicando que ela pode se tornar até mesmo árida no futuro.

\section{AgRADECIMENTOS}

Os autores agradecem ao Instituto Nacional de Meteorologia - INMET, e ao Departamento de Ciências Atmosféricas DCA, pelo fornecimento dos dados, e ao Conselho Nacional de Desenvolvimento Científico e Tecnológico - CNPq, pela oportunidade e auxílio para expansão profissional.

\section{LITERATURA CITADA}

Alves, J. M. D.; Kayano, M.T. Estudos preliminares da precipitação no Sul do Ceará durante a pré-estação das chuvas. Climanálise, v.6, p.42-50, 1991.

Arnell, N. W. Climate change and global water resources. Global Environmental Change, v.9, Supplement 1, p.S31-S49, 1999.

Back, A. J. Aplicação de análise estatística para identificação de tendências climáticas. Pesquisa Agropecuária Brasileira, v.36, n.5, p.717-726, 2001.

Barnes, S. L. A technique for maximizing details in numerical weather map analysis. Journal of Applied Meteorology, v.3, p.396-409, 1964.

Braganza, K.; Karoly, D. J.; Hirst, A. C.; Mann, M. E.; Stott, P.; Stouffer, R. J.; Tett, S.F.B. Simple indices of global climate variability and change: Part I - variability and correlation structure, Climate Dynamics, v.20, n.5, p.491-502, 2003.

Chan, S. C. Análise de distúrbios ondulatórios de leste sobre o Oceano Atlântico Equatorial Sul. São José dos Campos: INPE, 1990. 89p. Dissertação Mestrado

Cunha, L. V.; Oliveira, R.; Nunes, V. B. Impactos das alterações climáticas sobre os recursos hídricos de Portugal. Water Resources. v.137, p.201-222, 2002.

Hastenrath, S.; Heller, L. Dynamics of climatic hazards in northeast Brazil. Quarterly Journal Royal Meteorological Society, v.103, p.77-92, 1977.

Hertig, E.; Jacobeit, J. Downscaling future climate change: Temperature scenarios for the Mediterranean area. Global and Planetary Change, v.1, n.63, p.127-131, 2008.

IPCC - Intergovernmental Panel on Climate Change. Climate Change 2007: The physical science basis summary for policymakers. Geneva: IPCC. 2007. 21p.

Kendall, M. G. Rank correlation methods. London: Charles Griffin, 1975. 120p.

Ma, Z.; Kang, S.; Zhang, L.; Tong, L.; Su, X. Analysis of impacts of climate variability and human activity on streamflow for a river basin in arid region of northwest China. Journal of Hydrology, v.352, p.239-249, 2008.

Mann, H. B. Nonparametric tests against trend. Econometrica, v.13, p.245-259, 1945.

Marengo, J. A.; Valverde, M. C. Caracterização do clima no Século XX e Cenário de Mudanças de clima para o Brasil no Século XXI usando os modelos do IPCC-AR4. Revista Multiciência, v. 8, p.5-28, 2007.

Modarres R.; Silva, V. de P. R. Rainfall trends in arid and semiarid regions of Iran. Journal of Arid Environments, v.70, n.2, p.344-355, 2007.

Molion, L. C. B.; Bernardo, S. O. Uma revisão das chuvas no Nordeste brasileiro, Revista Brasileira de Meteorologia, v.17, n.1, p.1-10, 2002.

Nobre, C. A.; Molion, L. C. B. The climatology of drought and drought prediction. The impact of variations on agriculture, v.2, p.305-323, 1988.

Pimenta, M. T.; Santos, M. J.; Rodrigues, R. A. Susceptibilidade à desertificação. Revista Florestal, v.11, n.1, p.27-33, 1998.

Silva, V. de P. R. On climate variability in Northeast of Brazil. Journal of Arid Environments, v.58, n.4, p.575-596, 2004. 
Silva, V. de P. R.; Belo Filho, A. F.; Silva, B. B. da; Campos, J. H. B. C. Desenvolvimento de um sistema de estimativa a da evapotranspiração de referência. Revista Brasileira de Engenharia Agrícola e Ambiental, v.9, n.4, p.547-553, 2005.

Silva, V. de P. R.; Correia, A. A.; Coelho, M. S. Análise de tendência das series de precipitação pluvial do Nordeste do Brasil. Revista Brasileira de Engenharia Agrícola e Ambiental, v.2, n.1, p.111-114, 1998.

Silva, V. de P. R.; Sousa, F. A. S.; Cavalcanti, E. P.; Souza, E. P.; Silva, B. da B. da. Teleconnections between sea-surface temperature anomalies and air temperature in northeast Brazil. Journal of Atmospheric and Solar-Terrestrial Physics, v.68, n.1, p.781-792, 2006.
Sousa Júnior, I. F. A influência da urbanização no clima da cidade de Campina Grande, PB. Campina Grande: DCA, 2006. 94p. Dissertação Mestrado

Thornthwaite, C. W. An approach toward a rational classification of climate. Geographical Review, v.38, p.55-94, 1948.

Todisco, F.; Vergni, L. Climatic changes in Central Italy and their potential effects on corn water consumption. Agricultural and Forest Meteorology, v.148, n.1, p.1-11, 2008.

Yamazaki, Y.; Rao, V. B. Tropical cloudiness over the South Atlantic Ocean. Journal of the Meteorological Society of Japan, v.55, p.205-207, 1977. 